\title{
Bedside tracer gas technique accurately predicts outcome in aspiration of spontaneous pneumothorax
}

\author{
D G Kiely^, S Ansari, W A Davey, V Mahadevan, G J Taylor†, D Seaton
}

\begin{abstract}
Background-There is no technique in general use that reliably predicts the outcome of manual aspiration of spontaneous pneumothorax. We have hypothesised that the absence of a pleural leak at the time of aspiration will identify a group of patients in whom immediate discharge is unlikely to be complicated by early lung re-collapse and have tested this hypothesis by using a simple bedside tracer gas technique.
\end{abstract}

Methods-Eighty four episodes of primary spontaneous pneumothorax and 35 episodes of secondary spontaneous pneumothorax were studied prospectively. Patients breathed air containing a tracer (propellant gas from a pressurised metered dose inhaler) while the pneumothorax was aspirated percutaneously. Tracer gas in the aspirate was detected at the bedside using a portable flame ioniser and episodes were categorised as tracer gas positive ( $>1$ part per million of tracer gas) or negative. The presence of tracer gas was taken to imply a persistent pleural leak. Failure of manual aspiration and the need for a further intervention was based on chest radiographic appearances showing either failure of the lung to re-expand or re-collapse following initial reexpansion.

Results-A negative tracer gas test alone implied that manual aspiration would be successful in the treatment of $93 \%$ of episodes of primary spontaneous pneumothorax $(p<0.001)$ and in $86 \%$ of episodes of secondary spontaneous pneumothorax $(p=0.01)$. A positive test implied that manual aspiration would either fail to re-expand the lung or that early recollapse would occur despite initial reexpansion in $66 \%$ of episodes of primary spontaneous pneumothorax and $71 \%$ of episodes of secondary spontaneous pneumothorax. Lung re-inflation on the chest radiograph taken immediately after aspiration was a poor predictor of successful aspiration, with lung re-collapse occurring in $34 \%$ of episodes by the following day such that a further intervention was required.

Conclusions-National guidelines currently recommend immediate discharge of patients with primary spontaneous pneumothorax based primarily on the outcome of the post-aspiration chest radiograph which we have shown to be a poor predictor of early lung re-collapse. Using a simple bedside test in combination with the post-aspiration chest radiograph, we can predict with high accuracy the success of aspiration in achieving sustained lung re-inflation, thereby identifying patients with primary spontaneous pneumothorax who can be safely and immediately discharged home and those who should be observed overnight because of a significant risk of re-collapse, with an estimated re-admission rate of $1 \%$.

(Thorax 2001;56:617-621)

Keywords: pneumothorax; tracer gas; aspiration; discharge

National guidelines recommend that manual aspiration should be used as initial treatment for symptomatic spontaneous pneumothorax in the absence of life threatening features. ${ }^{1}$ They also imply that the state of lung expansion on the chest radiograph taken immediately after aspiration should be used to determine fitness for discharge the same day in primary spontaneous pneumothorax. Despite this, a recent survey of physicians working in acute medicine showed that two thirds of respondents were reluctant to discharge patients with primary spontaneous pneumothorax immediately, despite the post-aspiration radiograph indicating success. ${ }^{2}$ A test capable of showing closure of the pleural leak may provide a reliable guide to the success of aspiration when used in conjunction with the radiographic findings.

Having previously described a flame ionisation technique for detecting small concentrations of the hydrocarbon propellant gases used in standard pressurised metered dose inhalers, ${ }^{3}$ we adapted this method to detect pleural leaks during the aspiration of pneumothoraces and the results of a pilot study suggested that the technique may predict outcome. ${ }^{4}$ The present series tests this further by presenting our observations on the application of the tracer gas technique to 119 episodes of spontaneous pneumothorax.

\section{Methods}

STUDY POPULATION

Symptomatic patients with spontaneous pneumothorax who had been referred acutely to a general hospital serving a population of approximately 350000 were recruited over an 8 year period. These patients included the cohort 


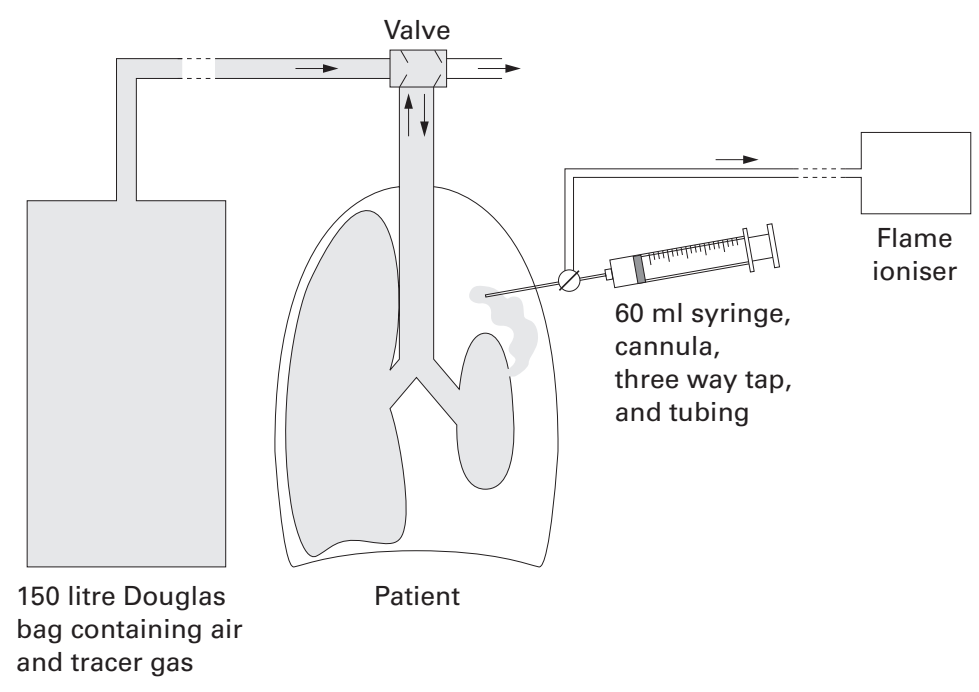

Figure 1 Diagrammatic representation of apparatus used to detect tracer gas (as previously described). ${ }^{4}$
The volumes of gas aspirated and the concentrations of tracer were recorded. Episodes were classified as "tracer gas positive" if the hydrocarbon concentration in the aspirated pneumothorax gas exceeded a threshold of $1 \mathrm{ppm}$ above the baseline reading. Episodes were otherwise "tracer gas negative". As the gut flora of some normal subjects produces methane, ${ }^{6}$ traces of which may pass from the patient's breath into the pneumothorax space, these subjects were routinely identified by using the flame ioniser to sample expired breath at the mouth, a baseline measurement then being made on the first syringeful of gas aspirated from the pneumothorax before breathing the tracer from the bag.

The protocol for performing a further intervention was based on radiographic appearances unless the patient became increasingly symptomatic during the procedure when the operator was at liberty to insert an intercostal chest drain without the need to perform a further chest radiograph. The radiographic size of the pneumothorax immediately after aspiration was classified as either "absent/shallow" or "similar size/larger". Shallow was defined as a significantly smaller pneumothorax with insufficient gas remaining to justify further intervention according to the size criterion above. A pneumothorax of similar/larger size on the post-aspiration radiograph indicated failed aspiration in which case an intercostal tube with underwater seal drainage was recommended. If the pneumothorax was absent/ shallow on the post-aspiration film, a further radiograph was taken not later than the following day. Again, if this radiograph showed that the pneumothorax had enlarged, a further pleural leak had occurred and re-aspiration or an intercostal tube was recommended if the original size criteria were met. When it was found that the pneumothorax was the same size or further diminished on this second radiograph, the pleural leak was taken to have sealed off and aspiration to have been successful. The patient was then discharged, if their general condition permitted, with arrangements for further outpatient radiographs to be taken at weekly intervals until resolution.

\section{STATISTICAL METHODS}

The categorical data were analysed using the $\chi^{2}$ test. Analysis of variance was used to compare the age differences between the groups with pneumothorax and the data are presented as mean (SD). All other analyses were made using the non-parametric Mann-Whitney U test and the data are presented as medians and interquartile ranges.

\section{Results}

Patients with primary spontaneous pneumothorax were significantly younger than those with secondary spontaneous pneumothorax (mean 35 (SD17) versus 53 (23) years, $\mathrm{p}<0.001)$. Male preponderance was more common in secondary than in primary spontaneous pneumothorax (8:1 versus $3: 1)$. Underlying lung disease in the 35 episodes of secondary spontaneous pneumothorax included 
Table 1 Accuracy of tracer gas technique in the detection of a persistent pleural leak requiring further procedure

\begin{tabular}{llllll}
\hline & $\begin{array}{l}\text { Sensitivity } \\
(\%)\end{array}$ & $\begin{array}{l}\text { Specificity } \\
(\%)\end{array}$ & PPV (\%) & NPV (\%) & $\begin{array}{l}\text { Prevalence of } \\
\text { ID/RA (\%) }\end{array}$ \\
\hline All groups & 95 & 55 & 67 & 92 & 50 \\
Primary SP & 95 & 59 & 66 & 93 & 45 \\
Secondary SP & 95 & 43 & 71 & 86 & 60 \\
\hline
\end{tabular}

$\mathrm{SP}=$ spontaneous pneumothorax $\mathrm{PPV}=$ positive predictive value $\mathrm{NPV}=$ negative predictive value; $\mathrm{ID}=$ intercostal chest drain; $\mathrm{RA}=$ re-aspiration.

chronic obstructive pulmonary disease $(n=12)$, asthma $(n=6)$, cystic fibrosis $(n=11)$, cryptogenic fibrosing alveolitis $(n=3)$, sarcoidosis, mesothelioma, tuberculosis, Marfan's syndrome (each $\mathrm{n}=2$ ), neurofibromatosis and empyema (each $\mathrm{n}=1$ ).

Fifty five of 84 episodes of primary spontaneous pneumothorax (65\%) and 28 of 35 episodes of secondary pneumothorax $(80 \%)$ gave a positive tracer gas test suggesting an ongoing pleural leak. There was a highly significant relationship for the whole group between a positive test and the need for a further procedure, and between a negative test and the success of aspiration $(p<0.001)$; these relationships held for both primary $(\mathrm{p}<0.001)$ and secondary spontaneous pneumothorax $(p<0.01)$. The sensitivity, specificity, negative and positive predictive values for the tracer gas test as an indicator of the need for a further intervention after initial aspiration are shown in table 1 .

Of the 29 patients with primary spontaneous pneumothorax who were tracer gas negative, two required a further intervention. In one patient, despite aspiration of $880 \mathrm{ml}$, the postaspiration radiograph showed no reduction in size of the pneumothorax and an intercostal chest drain was inserted. The remaining patient developed a further episode of chest pain and shortness of breath overnight and a repeat radiograph showed an increase in size of the pneumothorax compared with the initial film which indicated successful aspiration. The patient was subsequently successfully treated by re-aspiration and was tracer gas negative. Of the seven patients with secondary spontaneous pneumothorax who were tracer gas negative, one required a further intervention. This patient had cystic fibrosis and the postaspiration radiograph showed the pneumothorax to be significantly larger after the aspiration of $370 \mathrm{ml}$ of air. This event was successfully treated by intercostal tube drainage which was removed after 2 days. Twenty of the 28 patients with secondary spontaneous pneumothorax who were tracer gas positive required a further intervention. Of the 55 patients with primary spontaneous pneumothorax who were tracer gas positive, 36 required a further intervention.

By contrast, the chest radiograph taken immediately after aspiration did not reliably predict the success of this procedure. For primary spontaneous pneumothorax the radiograph taken immediately after aspiration showed the residual pneumothorax to be shallow or absent in $76 \%$ of all episodes $(n=64)$ but in the radiograph taken on the following day $30 \%(n=19)$ of these lungs had re-collapsed (table 2). For secondary spontaneous pneumothorax, $74 \%(n=26)$ were shallow or absent but $46 \%(n=12)$ re-collapsed (table 3$)$. All these re-collapses were treated with intercostal tube drainage except 10 that were managed by re-aspiration, although five ultimately required intercostal tube drainage.

During the period of outpatient follow up, two new episodes of spontaneous pneumothorax occurred in patients with primary spontaneous pneumothorax who had been successfully treated by aspiration and in whom the tracer gas test had been negative. These episodes, which were marked by the sudden onset of chest pain and dyspnoea, occurred four days and one week after the first episode. There were no further episodes in patients with secondary pneumothorax in which the tracer gas test had been negative.

As defined above, the protocol for performing a further intervention was based on radiographic appearance unless the patient became increasingly symptomatic during the procedure in which case the operator was free to insert an intercostal drain without the need to perform a further chest radiograph. Eight patients became symptomatic during aspiration and all were tracer gas positive. Of these, four had primary spontaneous pneumothorax

Table 2 Relationship between initial post-aspiration chest radiograph (CXR) and tracer gas status and the outcome of primary spontaneous pneumothorax

\begin{tabular}{lrlcl}
\hline & $n$ & No further intervention & Intercostal drain & Re-aspiration \\
\hline CXR improved; tracer gas -ve & 27 & $26(96 \%)$ & 0 & $1(4 \%)$ \\
CXR improved; tracer gas +ve & 37 & $19(51 \%)$ & $10(27 \%)$ & $8(22 \%)$ \\
CXR not improved; tracer gas -ve & 2 & $1(50 \%)$ & $1(50 \%)$ & 0 \\
CXR not improved; tracer gas +ve & 14 & 0 & $13(93 \%)$ & $1(7 \%)$ \\
CXR not performed; tracer gas +ve & 4 & 0 & $4(100 \%)$ & 0
\end{tabular}

CXR improved = significant reduction in size of the pneumothorax; CXR not improved = pneumothorax unchanged in size or larger.

Table 3 Relationship between initial post-aspiration chest radiograph (CXR) and tracer gas status and the outcome of secondary spontaneous pneumothorax

\begin{tabular}{lrlll}
\hline & $n$ & No further intervention & Intercostal drain & Re-aspiration \\
\hline CXR improved; tracer gas -ve & 6 & $6(100 \%)$ & 0 & 0 \\
CXR improved; tracer gas +ve & 20 & $8(40 \%)$ & $11(55 \%)$ & $1(5 \%)$ \\
CXR not improved; tracer gas -ve & 1 & 0 & $1(100 \%)$ & 0 \\
CXR not improved; tracer gas +ve & 4 & 0 & $3(75 \%)$ & $1(25 \%)$ \\
CXR not performed; tracer gas +ve & 4 & 0 & $4(100 \%)$ & 0
\end{tabular}

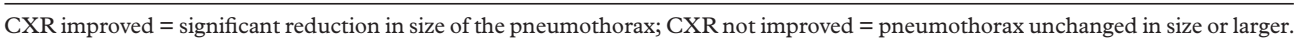


and had $3.001,3.481,4.001$, and 4.001 aspirated before insertion of drains which remained in situ for $7,5,6$, and 4 days, respectively. The four patients with secondary spontaneous pneumothorax had $0.301,0.721$, 0.781 , and 1.401 aspirated before insertion of intercostal drains which remained in situ for 10 days (until pleurectomy for persistent leak), 11 days (until pleurectomy for failure to expand), 2 days (patient died), and 12 days (until treated by pleurodesis).

Deviations from the protocol based on radiographic appearances occurred in nine episodes of primary and two episodes of secondary spontaneous pneumothorax, and all such deviations were in the direction of avoiding a further intervention. Of the primary spontaneous pneumothoraces, eight were tracer gas positive. The operators did not insert an intercostal drain in six of these episodes, despite the first post-aspiration radiograph showing that the pneumothorax was a similar size, as the patients were comfortable and did not wish to submit immediately to a further procedure. In four of these tracer gas positive episodes it was found that the pneumothoraces had enlarged by the following day, so three then required treatment by intercostal tube drainage while the other was re-aspirated and subsequently treated conservatively. The chest radiograph taken the following morning in the remaining two tracer gas positive episodes was the same size and an intercostal chest drain was then inserted. In a further two tracer gas positive episodes the pneumothorax was judged to be too small for further intervention on the immediate post-aspiration radiograph and, although the pneumothorax had increased slightly in size on the radiograph taken the following day, the operators judged that it was still too small to warrant intercostal tube drainage using the criteria previously outlined. Both of these patients subsequently achieved full resolution with no further events in the short term. The single tracer gas negative patient whose course did not adhere to the recommended protocol again showed only a small reduction in the size of the pneumothorax following aspiration, but the patient was comfortable and the size of the pneumothorax did not make an intercostal tube mandatory. This pneumothorax subsequently resolved with no further events in the short term.

There were two tracer gas positive episodes of secondary spontaneous pneumothorax in which a significant reduction in pneumothorax size on the immediate post-aspiration radiograph was followed by a slight increase in size of the pneumothoraces on the radiograph the next day. These were judged to be too small to justify further active intervention. The pneumothoraces in both these patients subsequently resolved with no further events in the short term.

\section{Discussion}

We have found that, in episodes of spontaneous pneumothorax, the lung is often re-inflated by manual aspiration only temporarily so that, although the chest radiograph taken immediately after aspiration may imply early success, it is insensitive at predicting sustained reinflation (tables 2 and 3). The significant strength of association between a positive tracer gas test and failure of the lung to re-expand at aspiration or early re-collapse following successful aspiration is likely to be due to a continued leak which, in the latter case, may be very small. The even stronger association between a negative tracer gas test and the subsequent success of aspiration in achieving sustained re-inflation implies accurate prediction of complete closure of the pleural leak (table 1).

How might such information be useful in the practical management of patients with spontaneous pneumothorax? The guidelines of the British Thoracic Society on the management of spontaneous pneumothorax recommend, for primary spontaneous pneumothorax, that the first radiograph taken after aspiration should be used to judge success and that the patient may be discharged if this image shows that the pneumothorax has either resolved or is small. ${ }^{1}$ Had the British Thoracic Society guidelines been applied to episodes of primary spontaneous pneumothorax in our observational series, 64 discharges would have been made on the same day as radiographically successful aspiration but readmission within 48 hours for intercostal tube drainage or further aspiration would have been necessary in $30 \%$ of these because of re-collapse (table 2).

If a decision to discharge had been based on the tracer gas technique and the radiographic findings, 27 episodes of primary spontaneous pneumothorax $(42 \%)$ in which aspiration produced lung re-expansion on the first film and which were tracer gas negative would have been immediately discharged. There would have been only one "false negative" patient needing re-admission in the next 48 hours $(1.2 \%$ of the cohort of 84 patients presenting with primary spontaneous pneumothorax). This "false negative" may reflect the natural propensity of spontaneous pneumothoraces to develop subsequent leaks rather than the ability of the tracer technique to detect a pleural leak at the time of the test, as this patient developed acute shortness of breath and chest pain following an initial film showing re-expansion but before we were able to perform a radiograph the following day as required under the protocol. The remainder (37 tracer gas positive episodes) would have been admitted overnight, discharge being possible in $51 \%(n=19)$ of them the following day when sustained lung expansion was confirmed on the later radiograph.

The British Thoracic Society guidelines recommend at least overnight observation for all patients with secondary spontaneous pneumothorax even if aspiration has apparently been successful on the immediate postaspiration radiograph. The data on secondary spontaneous pneumothorax in this series suggest that this advice is appropriate as $46 \%$ of apparently successful aspirations had recollapse of lung by the following day. This is in keeping with other published work. ${ }^{78}$ 
The study design was open rather than blinded in that the operator was aware of whether tracer gas was detected at the time of manual aspiration. This was due to ethical concerns that a patient with an ongoing pleural leak may be at increased risk of tension pneumothorax. The requirements for an intervention following aspiration were clearly defined and there is no evidence that the need for a further intervention was biased by the knowledge of tracer gas status. All eight patients who had an intercostal drain inserted without radiographic confirmation that the pneumothorax was larger or unchanged in size following aspiration undoubtedly required intercostal drainage for ongoing pleural leaks. This is based on the observation that the four patients with primary spontaneous pneumothorax had in excess of $3000 \mathrm{ml}$ aspirated (range 3000 $4000 \mathrm{ml}$ ) and had drains in situ for at least 4 days (range 4-7), while three of the four patients with secondary spontaneous pneumothorax required surgical intervention for ongoing leaks, the other patient dying with the drain in situ. All 11 (10 tracer gas positive) deviations from the strict radiographic criteria for a further intervention were made to avoid a further intervention, although six of these patients subsequently required a further intervention.

The findings of this study have shown that a significant number of patients admitted with both primary $(65 \%)$ and secondary spontaneous pneumothorax $(80 \%)$ have an ongoing pleural leak. It has been shown that these leaks do not all require intercostal tube drainage and are commonly too small to be detected on the immediate post-aspiration radiograph which is an insensitive predictor of outcome. The application of the tracer gas technique (particularly when used in combination with the immediate post-aspiration radiograph) permits more accurate risk stratification for patients susceptible to early lung re-collapse following aspiration, separating a group in which immediate discharge is likely to be uncomplicated from one in which re-collapse by the next day is more probable. Our current practice is to discharge patients who are tracer gas negative with primary spontaneous pneumothorax immediately if their chest radiograph shows a reduction in size of their pneumothorax, and to follow up these patients one week later, thereby reducing patient inconvenience and the economic implications of admission. All patients with primary spontaneous pneumothorax who are tracer gas positive are at risk of recollapsing their lungs and are observed at least overnight. An intercostal drain is only inserted if the chest radiograph the following day shows a significant increase in size as approximately one third of these patients will not re-collapse the lung, despite evidence of a small pleural leak at the time of manual aspiration. Patients with secondary spontaneous pneumothorax are all observed at least overnight with tracer gas positive patients identified on the ward as being at particularly high risk of requiring intercostal tube drainage.

We have found the technique to be well tolerated by patients and staff and applicable as a bedside test. The cost of the equipment (flame ioniser £2450) is not prohibitive. The phasing out of chlorofluorocarbons from metered dose inhalers ${ }^{9}$ does not affect the principle of the technique as we have found that the replacement hydrofluoroalkane propellants are also detectable by flame ionisation.

The authors are grateful to Dr Colin Ogilvie, Professor Anthony Seaton, and Sir Michael Stoker for their helpful comments and advice.

1 Miller AC, Harvey JE, on behalf of the Standards of Care Committee, British Thoracic Society. Guidelines for the management of spontaneous pneumothorax. BMf 1993;307:114-6.

2 Yeoh JH, Ansari S, Campbell IA. The management of spontaneous pneumothorax by hospital physicians and A \& E departments: a Welsh survey. Thorax 1998;53(Suppl 4):P112.

3 Coady TJ, Seaton D, Day A, et al. Measurement of exhaled chlorofluorocarbons. Lancet 1988;ii:1286-7.

4 Seaton D, Yoganathan K, Coady $\mathrm{T}$, et al. Spontaneous pneumothorax: marker gas technique for predicting outcome of manual aspiration. BMF 1991;302:262-5; 697 (erratum).

5 Seaton D. Pneumothorax. In: Seaton A, Seaton D, Leitch AG, eds. Crofton and Douglas's respiratory diseases. 5th ed. Oxford: Blackwell Scientific, 2000: 1182.

6 Cummings JH. The colon: physiology. In: Pounder RE, ed. Recent advances in gastroenterology. Vol 7. Edinburgh: Recent advances in gastroenterolog.

7 Bevelaqua FA. Management of spontaneous pneumothorax with small lumen catheter manual aspiration. Chest 1982;81:693-4

8 Archer GJ, Hamilton AAD, Upadhyay R, et al. Results of simple aspiration of pneumothoraces. Br F Dis Chest 1985; 79:177-82.

9 Partridge MR. Metered-dose inhalers and CFCs: what respiratory physicians need to know. Respir Med 1994;88:645- 